\title{
Image Stitching with Robust Principal Component Analysis
}

\author{
Wei Tao ${ }^{1,2}$, Zhang Yongxin ${ }^{3}$, Yuan Yating ${ }^{4}$ and Ji Xinsheng ${ }^{1}$ \\ ${ }^{1}$ National Digital Switching System Engineering \& Technological R\&D Center, \\ Zhengzhou Henan, 451000, China \\ ${ }^{2}$ Henan Institute of Engineering, Computer College, Zhengzhou Henan, \\ 451000, China \\ ${ }^{3}$ Luoyang Normal University, Luoyang Henan 471934, China \\ ${ }^{4}$ School of Information Science and Technology, Northwest University, Xi 'an \\ Shaanxi 710127, China \\ hnsqwt@163.com
}

\begin{abstract}
An image stitching algorithm based on the robustness of principal component analysis $(R P C A)$ is proposed in an effort to suppress the influence of noise in the image stitching quality. This algorithm represents high dimensional feature data by utilizing a lower dimensional linear subspace, and converts the image stitching problem into a principal component matrix matching problem. Through the use of a low rank matrix, the extraction of salient image characteristics is recovered and the noise interference is reduced during the enhancement process. Together, with the advantages of the RPCA algorithm, the algorithm improves the PSNR of the image while maintaining its strong matching ability. Experimental results show that the proposed scheme is able to significantly inhibit the noise and improve the stitching quality in comparison to the other existing stitching methods.
\end{abstract}

Keywords: image stitching; robust principal component analysis; feature points matching; saliency

\section{Introduction}

Image stitching can result in a high-resolution and wide-viewing angle image by finding a transformation matrix in the overlapping areas of two images. In recent years, the following image stitching methods, which have been based on features, including the Harris stitching algorithm [1], and the SIFT stitching algorithm [2], have been widely used due to their lower complexity and good applicability. However, images tend to contain outliers or acquire large sparse noise during the acquisition and transmission processes due to the effect of sensors and environmental factors. Thus, in the image stitching process, the noise is prone to being misjudged and extracted as feature points, which influences the accuracy of the image matching and the final stitching result. Additionally, image de-noising done using traditional algorithms, in which a suitable denoising algorithm will have to be selected according to the specific noise, results in its low adaptability as well as ineffective stitching image resolution; thus rendering the required effort useless. The removal of noise interference on the premise of ensuring the quality of image stitching [3] is significant in the formation of stitching image.

The SIFT stitching algorithm is one of the main stream algorithms [4]. It is invariant to image scale transform and image rotation, however, it is still limited in the elimination of noise interference. Many recent studies have focused on the improvement of the SIFT algorithm. One such study proposed the PCA-SIFT algorithm[5], whose operation efficiency is improved by reducing the dimension of the feature descriptor, however, its 
matching accuracy is lower in comparison to the SIFT algorithm. Another example includes the Speeded Up Robust Features (SUFT) algorithm [6]. This algorithm performs stitching using an integral image; however, the resulting image is not ideal due to the noise interference. These studies indicate that most of the existing image stitching algorithms are designed for environments without noise. It is difficult to make an accurate judgment of the image feature points in an environment with noise, which leads to a decline in the stitching image quality. The robust principal component analysis algorithm (RPCA) has been proven to be an effective method in representing image data in low dimensional linear subspace and has been successfully applied in many fields. In addition to its strong robustness to noise, it is conducive to the extraction of salient features in the source image and ensures the quality of stitching image.

In this paper, we propose a novel image stitching scheme that is different from the methods described above. This method is implemented in an RPCA decomposition domain [7]. RPCA is a very important method of low-rank matrix recovery (LRMR) [8]. The potential application of RPCA in image fusion has been investigated and was shown to have consistently good fusion results, which is useful for stitching [9]. The method proposed in this paper differs in that this method is based on sparse features for image stitching. The principal and sparse matrices of the source images are obtained using RPCA decomposition. Correct image matching of the source images is obtained by computing the salient feature of the corresponding sparse matrix. The proposed method can significantly reduce the noise as well as enhance the ability to extract the image details from the source images.

The rest of the paper is organized as follows: In Section 2, the basic idea of RPCA is briefly described, followed by the proposal of the new method based on RPCA for image stitching in Section 3. In Section 4, extensive simulations are performed in order to evaluate the performance of the proposed method. In addition, several experimental results are presented and discussed. Finally, the concluding remarks are given in Section 5.

\section{Related Work}

\subsection{Robust Principal Component Analysis}

The traditional Principal Component Analysis (PCA) algorithm is able to perform data dimension reduction by utilizing the linear subspace model. However, this algorithm only takes the structures of adjacent samples into consideration and has a very poor robustness when influenced by factors including noise, and light. Here, we propose a PCA with robustness in an effort to solve this issue. RPCA [10] has the capability to determine the best way to represent original data using as few variables as possible in a noisy environment, for example, the large data matrix $D \in i^{M \times N}$ is decomposed into a low rank matrix $A \in i^{M \times N}$ and a sparse matrix $E \in i^{M \times N}$. Where $A$ and $E$ are unknown, as shown in the following:

$$
\min _{A, E}\|A\|_{*}+\lambda\|E\|_{1.1}, \text { s.t. } A+E=D
$$

Where $\|\cdot\|_{F}$ is the Frobenius norm. This formula is effectively solved through the evaluation of the Singular Value Decomposition (SVD) in matrix $D$. However, this method is no longer applicable when $E$ is a sparse matrix and a lot of noise is present. The task of recovering the low rank matrix is transformed into a double optimization, as follows: 
$\min _{A, E}\left(\operatorname{rank}(A),\|E\|_{0}\right)$, s.t. $A+E=D$

The problem is simplified by adopting the compromise factor $\lambda$ in order to convert formula (2) into a single optimization problem:

$\min _{A, E}\left(\operatorname{rank}(A)+\lambda\|E\|_{0}\right)$, s.t. $A+E=D$

The optimized result of (3) is still an NP-hard problem, thus, the objective function needs to be relaxed and converted into a convex optimization problem:

$\min _{A, E}\|A\|_{*}+\lambda\|E\|_{1.1}$, s.t. $A+E=D$

Typically, the value of $\lambda$ is a positive weighting parameter and $\lambda=1 / \sqrt{\max (m, n)}$. The optimization of the type (4) problem is called the robust principal component analysis. The source image is a Lena image, which added a density of pepper-salt noise of 0.05 and is shown below in Figure 1.

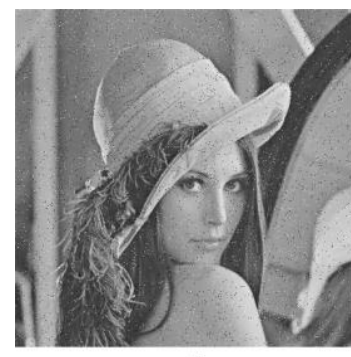

source image

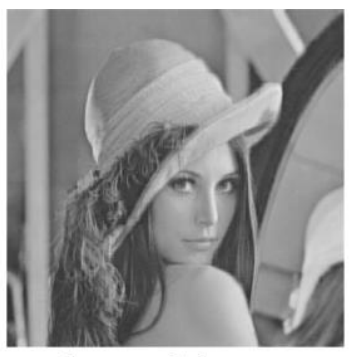

low rank image

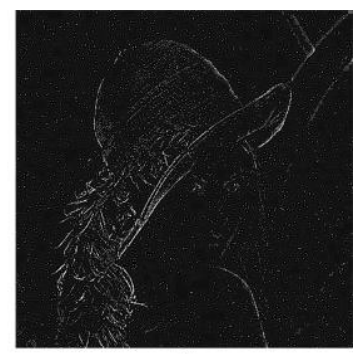

sparse image

Figure 1. Decomposition of Multi-Focus Images 'Lena' using RPCA. (a) Source Image $D$, (b) Principal Matrix $A$, (c) Sparse Matrix $E$

Identifying the feature points is the core of image stitching; the existence of noise affects the accurate judgment of the feature points and reduces the quality of stitching. Therefore, one cannot ignore the influence of noise on stitching image. Extracting feature points from images that contain noise using traditional stitching algorithms is difficult. High-dimensional data can be represented by means of a lower dimensional linear space using RPCA, on the premise of guaranteeing the image effect, and decreasing the impact of the noise [11]. Therefore, the RPCA algorithm in a low-rank matrix recovery of the source stitching image is adopted in this paper, which is used to improve the effect of image stitching under the influence of noise. To ensure the quality of the stitching image and to enhance the robustness of the algorithm in regards to the presence of noise, while reducing false match logarithms at the same time, an image stitching algorithm based on RPCA using SIFT features extracted from the sparse component of the source images is proposed in this paper.

\section{Proposed Method}

The RPCA can extract the image saliency and improve noise robustness according to the above analysis. Coupled with the classical SIFT image stitching algorithm, a new image stitching algorithm based on RPCA is proposed; the framework of which is depicted in Figure 2. 


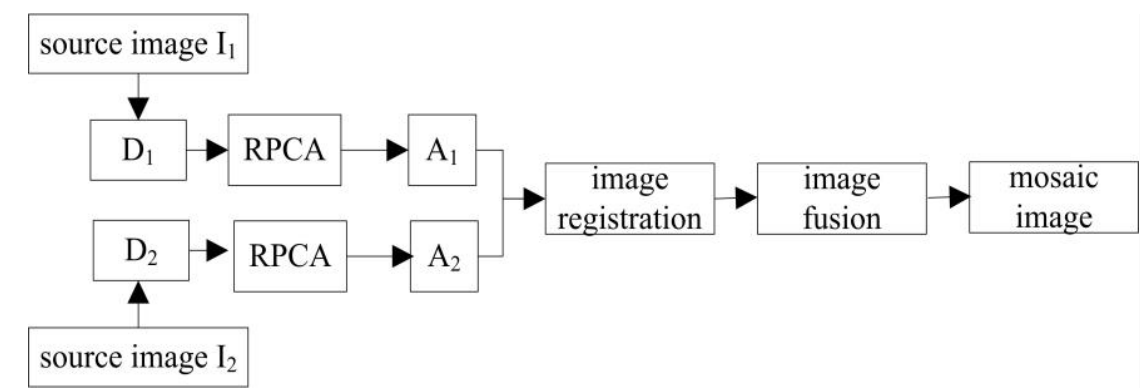

Figure 2. Block Diagram of Proposed Images Stitching Framework

For simplicity, it is assumed that there are only two source images, $I_{1}$ and $I_{2}$. However, the rationale behind the proposed scheme is also applicable to the stitching of more than two images. The stitching algorithm consists of the following four steps:

Step 1: image acquisition: The source images $I_{1}$ and $I_{2}$ are acquired by means of a fixed-point rotation or the movable shooting along the direction perpendicular to the optical axis of the camera. After the vector transformation, the corresponding data matrix $D_{1}$ and $D_{2}$ is formed;

Step 2: image processing by the RPCA algorithm: The data matrix $D_{1}$ and $D_{2}$ experiences RPCA decomposition and the vector transformation, resulting in the principal component matrix $A_{1}$ and $A_{2}$ corresponding to the source images, and sharing the same size with the source images $I_{1}$ and $I_{2}$. In summary, in the RPCA decomposition domain of source images, the principal component matrix is extracted (salient features of the image), enabling the processed images to maintain maximum image saliency while effectively eliminating noise interference.

Step 3: image registration: SIFT image registration methods are applied [12] to the salient features $A_{1}$ and $A_{2}$ that were obtained in Step 2. In order to improve the accuracy of image matching, the RANSAC algorithm is used in order to evaluate and refine the transformation matrix.

Step 4: image fusion: The simple and rapid weighted smoothing algorithm (WSA) [13] is used in order to deal with the seams and the adjustable factor $k=d 1 /(d 1+d 2)$ [14]. Where $d_{1}$ and $d_{2}$ refer to the distance from the points in the overlapping area from its left and right side.

Step 5: fusion images output: Output the complete stitching images.

\section{Experimental Results}

The validity of the proposed method is evaluated using an experiment that is performed on a set of 2 pairs of source images differing in content, shooting times, views and light intensity, as is shown in Figure 3. Salt and pepper noise are added to the source images, Poisson noise and Gaussian noise, respectively. $(\sigma=0.02)$. The two pairs are grayscale images with a size of $411 \times 512$ and $240 \times 180$ pixels, respectively. Experiments are conducted using Matlab in the Windows environment on a computer with Intel Xeon X5570 and 48G of memory. Current existing image stitching methods are also used on the same set of source images for the purpose of comparisons with the proposed method. The other existing methods used include SIFT, PCA-SIFT, and SUFT. The algorithm in this paper is implemented using MATLAB language in a MATLAB R2010a environment. In order to quantitatively compare the performance of proposed method, as well as the existing methods mentioned above, three metrics are used to evaluate the fusion performance. These metrics include: (i) the Peak Signal to Noise Ratio (PSNR), which reveals the presence of the noise in the stitching images [15]. A larger PSNR value signifies a better stitching result. 


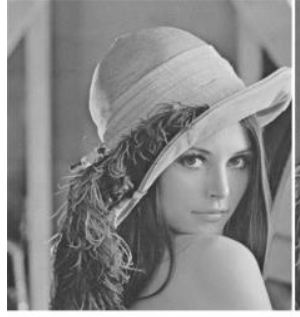

(a)

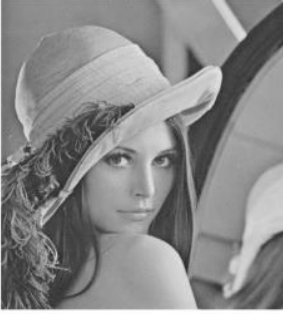

(b)

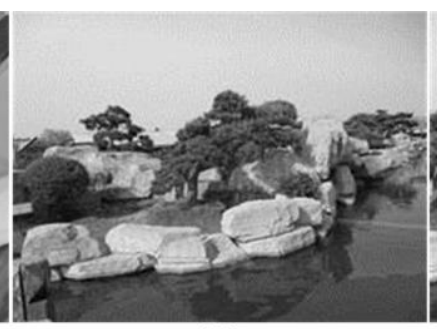

(c)

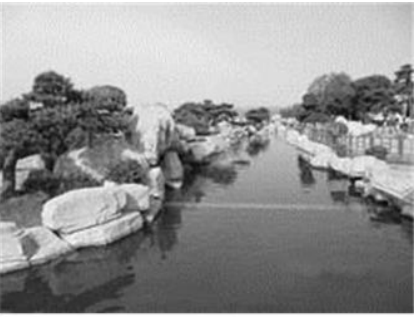

(d)

Figure 3. Source Images. (a) Left Image 'Lena', (b) Right Image ' Lena', (c) Left Image 'Lake', (d) Right Image 'Lake'

\subsection{Qualitative Analysis}

The stitching images obtained using different methods in different noise environment are shown in Figures 4 (a-d), Figures 5 (a-d), and Figures 6 (a-d). The stitching images obtained using different methods in with different Gaussian noise intensities are shown in Figures 7 (a-d), and Figures 8 (a-d). The stitching images obtained using the other fusion methods demonstrate an obvious black gap, deformation and a visible sift. As is seen in Figure 4 (a), Figure 5 (a), and Figure 6 (a), these images have been significantly affected by noise that an obvious black gap appears. The low resolution is apparent in Figure 4 (b), Figure 5 (b), and Figure 6 (b), due to the interference of noise, however the visual quality of Figure 4 (b), Figure 5 (b), and Figure 6 (b), are all better in comparison to Figure 4 (a), Figure 5 (a), and Figure 6 (a). These results demonstrate that the stitching method based on PCA-SIFT is able to suppress certain noise. The stitching method based on SUFT is not robust to noisy conditions. A certain deformation and a visible sift are both apparent in Figure 4 (c), Figure 5 (c), and Figure 6 (c). The proposed stitching algorithm has the ability to extract the salient features of the source images. The detailed information, such as the edge of the hat and the texture of the stone are apparent in Figure 4 (d), Figure 5 (d), and Figure 6 (d). The declining image quality of the stitching images with the subsequent increase in noise intensity is obvious in Figures 7 (a-d), and Figures 8 (a-d). The quality of Figure 7 (a-d), is better in comparison to Figure 8 (a-d). An obvious gap appears in Figure 7 (a), Figure 8(a), and Figure 8(c). The quality of Figure 8 (d), and Figure 8 (d), are relatively stable. These results demonstrate that the proposed method is capable of stitching the source images and effective in suppressing the noise.

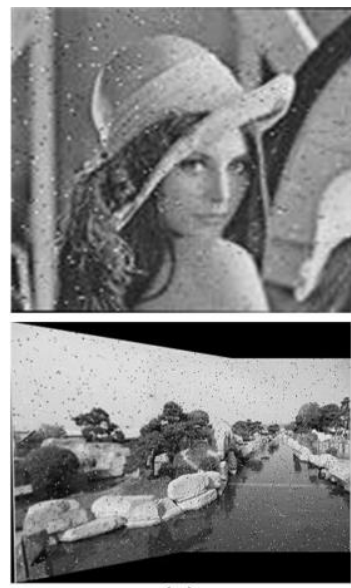

(a)

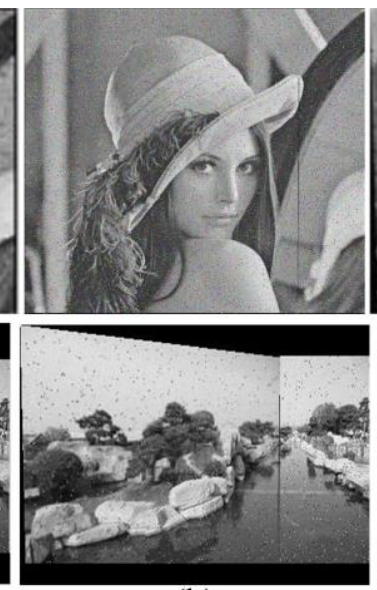

(b)

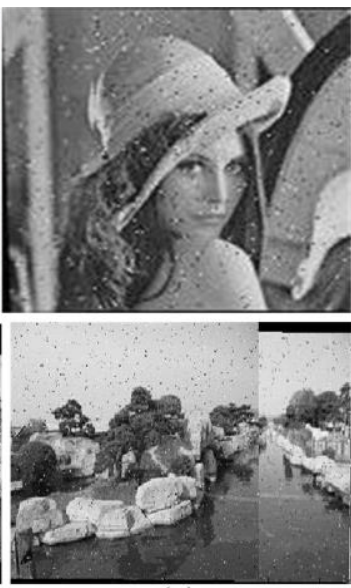

(c)

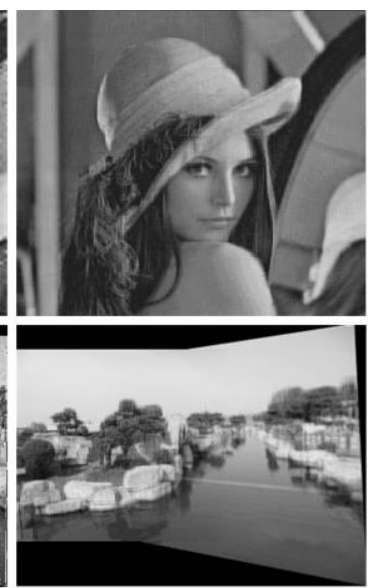

(d)

Figure 4. Stitching Images Obtained by SIFT (a), PCA-SIFT (b), SUFT (c) and the Proposed Method (d) in Salt \& Pepper Noise Environment 


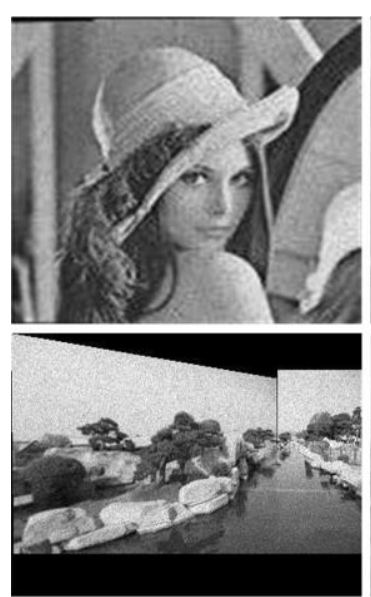

(a)

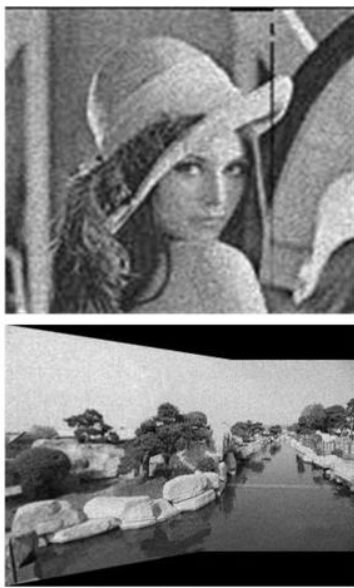

(b)

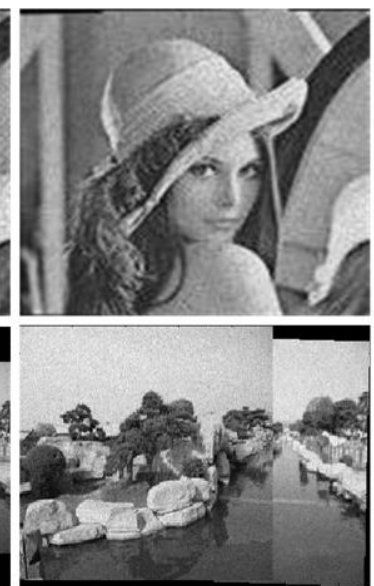

(c)

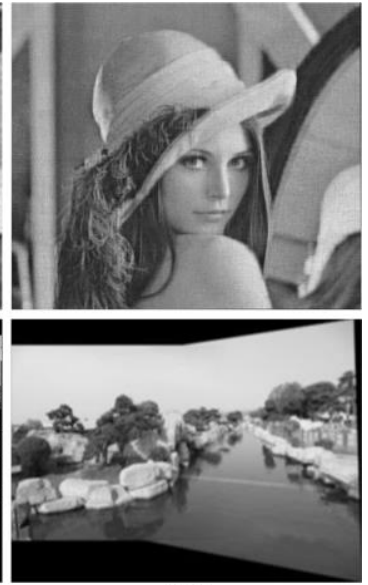

(d)

Figure 5. Stitching Images Obtained by SIFT (a), PCA-SIFT (b), SUFT(c) and the Proposed Method (d) in Poisson Noise Environment

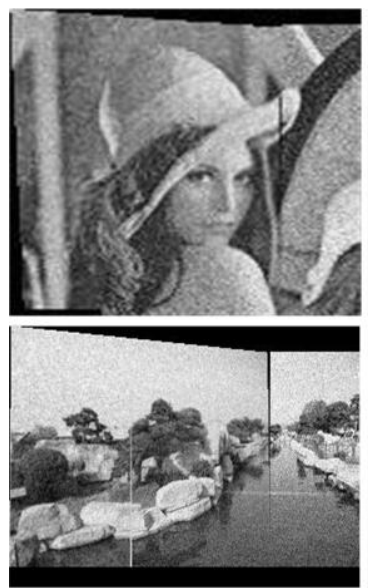

(a)

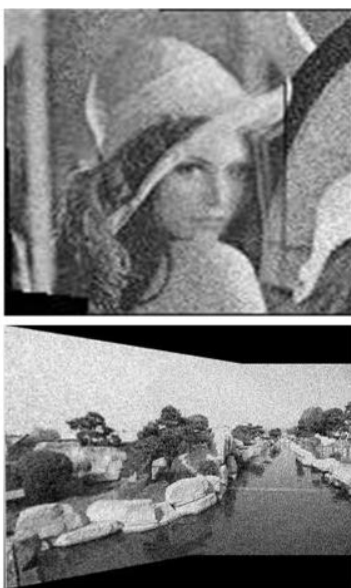

(b)

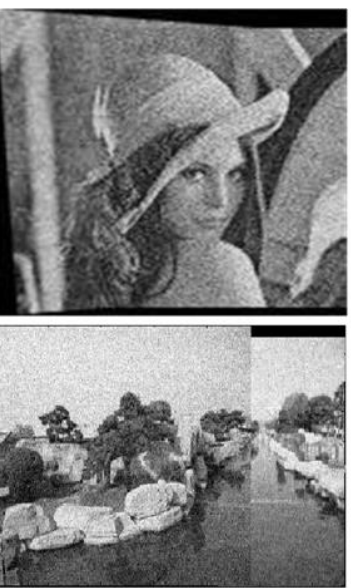

(c)

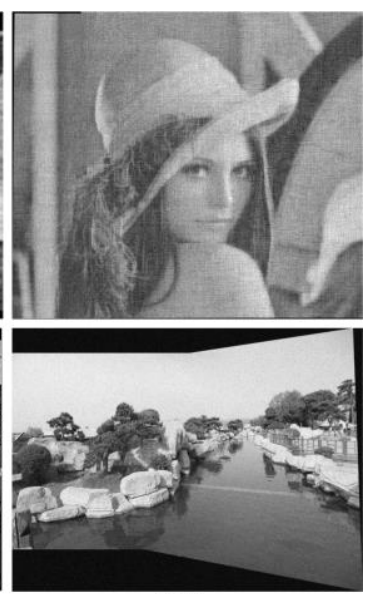

(d)

Figure 6. Stitching Images Obtained by SIFT (a), PCA-SIFT (b), SUFT(c) and the Proposed Method (d) in Gaussian Noise Environment $(\sigma=0.02)$

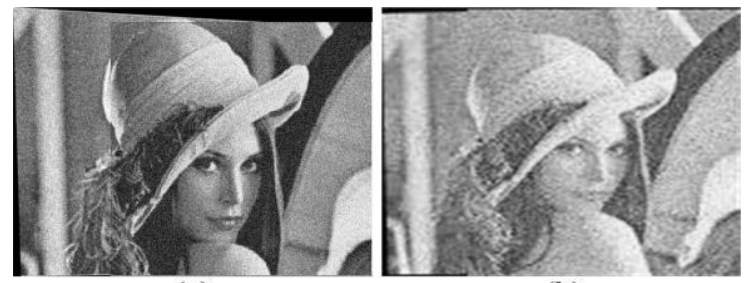

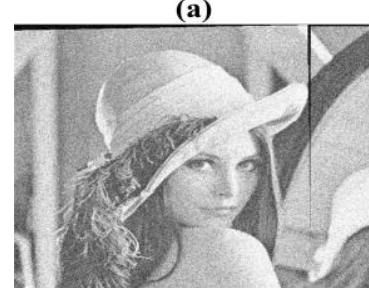

(c) (b)

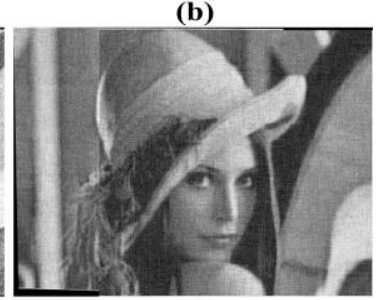

(d)

Figure 7. Stitching Images Obtained by SIFT (a), PCA-SIFT (b), SUFT(c) and the Proposed Method (d) in Gaussian Noise Environment $(\sigma=0.2)$ 


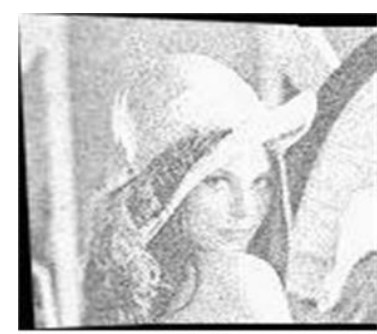

(a)

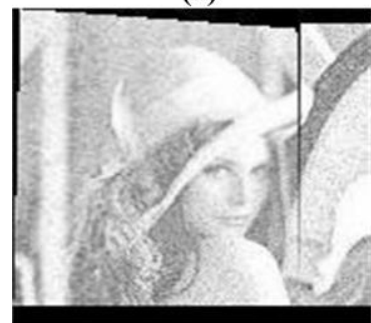

(c)

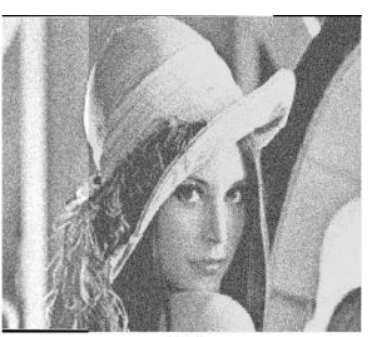

(b)

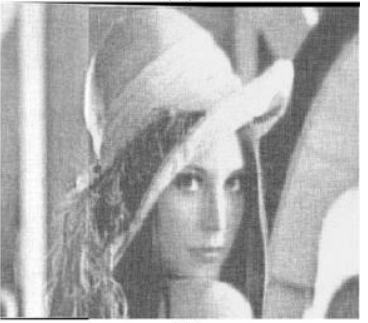

(d)

Figure 8. Stitching Images Obtained by SIFT (a), PCA-SIFT (b), SUFT(c) and the Proposed Method (d) in Gaussian Noise Environment $(\sigma=0.4)$

\subsection{Quantitative Analysis}

For a quantitative comparison, the PSNR of stitching images that was obtained using the different methods in different noise environments are shown in Table 1. The proposed method was shown to result in higher PSNR values when compared to the other methods. The stitching method based on SIFT is rated better than that of the PCA-SIFT and SUFT. The stitching method based on the PCA-SIFT was shown to result in lower PSNR values in comparison to the other methods. The PSNR of the stitching image "Lena" obtained using the different methods in different environments of varying Gaussian noise intensities are shown in Table 2. The proposed method was shown to result in higher PSNR values in comparison to SIFT. It is apparent that the PSNR values are reduced with the subsequent increase in the intensity of noise. The proposed method significantly outperforms the stitching method based on SIFT when the noise intensity value is 0.2 or 0.4 , however, does not have any competitive advantage when the noise intensity value is 0.02 .

Table 1. The PSNR of Stitching Images Obtained by Different Methods in Different Noise Environments

\begin{tabular}{|c|c|c|c|c|c|c|c|c|}
\hline \multirow{2}{*}{$\begin{array}{c}\text { Noise } \\
(\sigma=0.02)\end{array}$} & \multicolumn{4}{|c|}{ Lena } & \multicolumn{4}{c|}{ Lake } \\
\cline { 2 - 10 } & SIFT & PCA-SIFT & SUFT & Proposed & SIFT & PCA-SIFT & SUFT & Proposed \\
\hline Salt \& Peper & 14.0761 & 11.3568 & 12.9658 & $\mathbf{1 5 . 7 3 6 3}$ & 17.9059 & 16.2354 & 16.5656 & $\mathbf{2 0 . 8 9 8 9}$ \\
\hline Poisson & 25.3853 & 14.0001 & 14.5628 & $\mathbf{2 7 . 3 5 0 5}$ & 17.0442 & 15.6589 & 16.0003 & $\mathbf{1 8 . 0 1 8 4}$ \\
\hline Gaussian & 19.5187 & 17.2312 & 18.0256 & $\mathbf{1 9 . 7 5 0 6}$ & 15.4066 & 13.2577 & 14.2537 & $\mathbf{1 6 . 4 7 4 1}$ \\
\hline
\end{tabular}

Table 2. The PSNR of Stitching Image "Lena" Obtained by Different Methods in Different Intensity Gaussian Noise Environments

\begin{tabular}{|c|c|c|}
\hline Intensity & SIFT & Proposed \\
\hline 0.02 & 19.5187 & $\mathbf{1 9 . 7 5 0 6}$ \\
\hline 0.2 & 11.4419 & $\mathbf{1 4 . 6 5 8 8}$ \\
\hline 0.4 & 8.5759 & $\mathbf{9 . 2 0 0 0}$ \\
\hline
\end{tabular}




\section{Conclusion and Future Work}

This paper proposes a novel image stitching method based on RPCA, which uses sparse features to improve the stitching quality. Evaluations of the performance have demonstrated that the proposed method results in a better stitching image and is able to significantly inhibit the noise. However, the proposed method has a high computational cost for the matrix decomposition, and only has a general performance in a low noise environment. In the future, we will consider extending the developed method to the stitching of remote sensing images.

\section{Acknowledgements}

The work is supported by the National Nature Science Foundation of China (No. 61502219), Postdoctoral Science Foundation of China (No. 2015M582697), the Natural Science Basic Research Plan in Henan Province of China (No. 2012JQ1012), the Innovative Talents of Science and Technology Support Plan in Henan Province of China (No. 17HASTIT024) and Henan Province Basic and Cutting-Edge Technology Research Project (No. 142300410374).

\section{References}

[1] S. Pravenaa and R. Menaka, "A Methodical Review on Image Stitching and Video Stitching Techniques", International Journal of Applied Engineering Research, vol. 11, no. 5, (2016).

[2] T. Shaikh and A. Patankar, "Multiple Feature Extraction Techniques in Image Stitching", International Journal of Computer Applications, vol.123, no.15, (2015).

[3] L. Zhu, Y. Wang and S. Liu, "A fast image stitching algorithm based on improved SURF", Proceedings of Tenth IEEE International Conference on Semantic Computing, (2014), Yunnan, China, November $15-16$.

[4] J. Zhang, G. Chen and Z. Jia. "An Image Stitching Algorithm Based on Histogram Matching and SIFT Algorithm", International Journal of Pattern Recognition and Artificial Intelligence, (2016).

[5] T. Tao, "Small celestial body image feature matching method based on PCA-SIFT", Proceedings of Control Conference, , Hangzhou, China, (2015), July 28-30.

[6] S. Govindaraju and G. Kumar, "A Novel Content Based Medical Image Retrieval using SURF Features", Indian Journal of Science and Technology, vol. 9, (2016).

[7] Y. Zhang and L. Chen, "Multi-Focus Image Fusion Based on Sparse Decomposition" International Journal of Signal Processing, Image Processing and Pattern Recognition, vol.8, no. 2, (2015).

[8] E. Candes, X. Li, Y. Ma, J. Wright, "Robust principal component analysis?", JACM, vol. 58, no. 3, (2011).

[9] T. Wan, C. Zhub and Z. Qin, "Multi-focus Image Fusion Based on Robust Principal Component Analysis", Pattern Recognition Letters, vol. 34, no. 9, (2013).

[10] J. Wright, A. Ganesh, S. Rao and Y. Ma, "Robust principal component analysis: Exact recovery of corrupted low-rank matrices via convex optimization", Proceedings of Neural Information Processing System, Bangkok, Thailand, (2009) December 1-5.

[11] Y. Zhang and L. Chen, "Multi-focus image fusion based on sparse features" International Journal of Signal Processing, Image Processing and Pattern Recognition, vol.7, no. 2, (2014).

[12] F. Wang and H. You, "Adapted anisotropic Gaussian SIFT matching strategy for SAR registration", IEEE Geoscience and Remote Sensing Letters, vol.12, no. 1, (2015).

[13] J. Qian, L. Wang and Y. Zhang, "An adaptive weighted spatial smoothing algorithm utilizing virtual beam forming", IEEE International Radar Conference, IEEE, (2005).

[14] L. Huang and C. Chen, "Adaptive registration algorithm of color images based on SURF" Measurement, vol. 66, (2015).

[15] Q. Guo and C. Zhang, "An efficient SVD-based method for image de-noising", IEEE transactions on Circuits and Systems for Video Technology, vol. 26, no. 5, (2016).

\section{Authors}

Tao Wei, is currently pursuing a PhD degree at National Digital Switching System Engineering \& Technological R\&D Center, Zhengzhou, China. His research interests include image processing and pattern recognition. 
Yongxin Zhang, is an associate professor at Luoyang Normal University, Luoyang, China. His research interests include image fusion and pattern recognition.

Yating Yuan, is currently pursuing an master degree at Northwest University, Xi'an, China. His research interests include image fusion and pattern recognition.

Xinsheng Ji, is a professor at National Digital Switching System Engineering \& Technological R\&D Center, Zhengzhou, China. His research interests include image processing and pattern recognition. 
International Journal of Signal Processing, Image Processing and Pattern Recognition Vol. 9, No. 12, (2016) 